\title{
Complex segregation analysis of Gerbera flower colour
}

\author{
KENNETH R. TOURJEE*, JAMES HARDING \& THOMAS G. BYRNE \\ Department of Environmental Horticulture, University of California, Davis, CA 95616, U.S.A.
}

\begin{abstract}
The distribution of hue (CIELAB colour notation) classes among flowers of the Davis population of gerbera (Gerbera jamesonii H. Bolus ex Hooker) appears bimodal. This suggests that the genetic control of hue is determined by the segregation of a gene with large effect modified by additional genes with smaller effects. Complex segregation analysis (CSA), routinely employed in human genetic epidemiology, was used to study both qualitative and quantitative variation. CSA applies pedigree analysis through the consideration of transmission probabilities to optimize likelihood functions of various genetic models. Applying this technique to study flower hue of a sample representing generations 14, 15 and 16 of the Davis population, allowed identification of a putative dominant major gene with genotypic values for the dominant homozygote, heterozygote and recessive homozygote of 32,32 and 71 degrees, respectively. This corresponds to the modes of the hue frequency distribution for the population. The putative major gene represents 0.66 of the total variation. The residual parent-offspring correlation $\left(\rho_{\mathrm{po}}=0.2\right)$ measures the genetic contribution to the remainder of the variance.
\end{abstract}

Keywords: hue, major genes, pedigree analysis, quantitative traits, regressive models, statistical genetics.

\section{Introduction}

Flower colour in gerbera is determined by pigments of the flavonoid and carotenoid pathways (Valadon \& Mummery, 1967; Asen, 1984) but, because of the difficulty in defining phenotypic colour classes (Vandoni, 1977), its variation has not been described successfully by Mendelian segregation ratios. Random mating populations of gerbera have a pattern of continuous variation for flower colour suggesting the use of biometrical genetic analysis to elucidate modes of inheritance. However, the distributions for the colour attributes hue and value in the Davis population of gerbera appear bimodal (Tourjee et al., 1993), consistent with the presence of a gene with major effect segregating in a background of polygenic variation (Falconer, 1989; Hoeschele, 1988). Therefore, the conventional methods of segregation analysis and biometrical analysis are not appropriate for analysing flower colour variation in the Davis population.

The genetics underlying bimodal distributions has been studied by a number of researchers. Harris (1910) demonstrated a bimodal distribution for lamina

\footnotetext{
*Correspondence.
}

number per inflorescence in Syndesmon thalictroides Hoffmgg. and reviewed the literature concerning bimodal variation. Sinnott (1932) described a bimodal frequency distribution for a gourd-shape index in a population of Cucurbita pepo L. He concluded that the distribution derived from the segregation of both major and minor factors, and described such populations as complex. Resistance to the woolly apple aphis (Eriosoma lanigerum Hausmn.) in the apple cultivar 'Northern Spy' (Malus domestica Borkh.) was described as an example of complex inheritance because it was believed that both major gene and polygenic effects were present (Crane et al., 1936). Powers (1950) discussed the roles of major genes and polygenes in determining the weight per locule in tomato hybrids (Lycopersicum esculentum Mill.). Elkind et al. (1990) discussed the interaction of single genes with polygenes and their effect on tomato fruit softness. The recent literature concerning major gene and polygenic models was reviewed by Mitchell-Olds \& Bergelson (1990).

Complex segregation analysis (CSA), first suggested by Edwards (1960), provides a framework to investigate complex variation and permits the interpretation of genetic data in terms of both major and minor gene 
effects. Various applications of CSA have been developed and employed successfully in human genetic epidemiology (Elston \& Rao, 1978; Iselius, 1988). It is applicable also to any diploid cross-pollinated crop for which it is feasible to maintain a pedigree (for example gerbera, almonds (Prunus dulcis (Mill.) D.A. Webb), carnations (Dianthus caryophyllous L.), onions (Allium cepa L.) and red raspberries (Rubus idaeus L.), etc.).

The purpose of this study was to investigate the genetic basis of flower colour variation in the Davis population of gerbera through complex segregation analysis of hue, and to determine the relative effects of major loci and minor genes as contributing factors to this variation. A comparison of several genetic models is made.

\section{Materials and methods}

\section{Gerbera population and colour measurements}

Gerbera is a member of the Compositae and is a heterozygous, diploid, cut-flower crop. The Davis population has been randomly mated and grown as an annual in the same greenhouse for 16 generations. Plants were randomly assigned to locations within the greenhouse. In generations 14,15 and 16,40 parents have been selected from about 400 plants and intermated via a disconnected factorial mating design (NCII), with 10 parents per set. In these generations selection has been based on an index that has included yield of flowers and flower dry weight; selection has not been practised on flower colour. This population and its colour traits have been described more fully elsewhere (Yu et al., 1991; Tourjee et al., 1993).

Colour measurements of CIELAB 1976 hue angle, chroma and value (McGuire, 1992) were obtained using the Minolta (Ramsey, New Jersey) CR-200 chromameter. It is a tristimulus colour analyser for measuring the reflective colours of surfaces. The CR200 has an $8 \mathrm{~mm}$ diameter aperture and uses diffuse illumination from a pulsed xenon arc lamp with a 0 degree viewing angle. Measurements were made using CIE illuminant $\mathrm{C}$; the procedure is described in Tourjee et al. (1993). Colour data were collected on individuals from generations 14,15 and 16 of the Davis population.

Normal probability plots provide a convenient diagnostic tool for assessing distributional assumptions about data (Chambers et al., 1983). They yield more information than frequency distributions because they directly compare a sample's distribution to a normal probability function. These graphs are constructed by plotting the normal quantiles of a data set against its empirical quantiles. Normal quantiles are the normal scores for a data set; Minitab (Penn State University, University Park, PA) provides them as output from the 'Nscore' command. Empirical quantiles are the raw data scores. Normal probability plots of data sampled from a normal distribution will form a straight line. Systematic departures from a straight line pattern indicate that the theoretical distribution and data distribution do not match (for example, a data distribution skewed to the left is revealed as a curve of decreasing slope with increasing normal score, and data from a mixture of distributions produce a sigmoidal-shaped curve). Hoeschele (1988), demonstrated the use of normal probability plots to detect major genes in quantitative traits.

\section{CSA theory}

CSA derives information from the structure of pedigrees instead of comparing family means as in the Elkind \& Cahaner (1986) mixed model. Therefore, it is suitable for both observational and experimental studies. Using the existing pedigree structure of a breeding programme obviates the need to construct a population suitable for experimental analysis as required by the Elkind-Cahaner model.

Pedigree analysis was developed by Elston \& Stewart (1971) and further refined by Cannings et al. (1978); its efficacy and robustness was demonstrated by Go et al. (1978). A recursive procedure is used to peel off certain members of a pedigree successively and, using transition matrices (Smith, 1976), collapse the information derived from them onto a subset of the remaining members. This procedure uses all of the information from an extended pedigree rather than the limited amount available for conventional segregation analysis of sibship data. However, analysis of large extended pedigrees may not be efficient because many branches of the pedigree may not be segregating for the gene of interest. Therefore, pedigrees of intermediate size (namely 9-15 individuals) often have more statistical power to detect segregating genes than larger extended pedigrees (Burns et al., 1984).

Regressive models (Bonney, 1984) define residuals from major gene effects (i.e. $y=x-\mu_{\text {g }}$; where $x$ is the phenotypic measure of an individual and $\mu_{\mathrm{g}}$ is the appropriate genotypic mean) that are treated as dependent random variables. Briefly, these models use pedigree analysis to adjust the residuals of each individual in a pedigree by a set of explanatory variables, including major gene effects and the phenotypes of ancestors, to produce a new set of uncorrelated variables. The general regressive model for pedigrees of size $n$ is:

$z_{i}=y_{i}-\left(b_{i, 1} y_{1}\right)-\left(b_{i, 2} y_{2}\right)-\ldots-\left(b_{n, n-1} y_{n-1}\right)$, 
where $z_{i}$ is the adjusted residual of an individual in a pedigree (indexed $i=1$ to $n$ ), $y_{i}$ is the residual from the major genotype (RMG) and the $b$ s are the regression coefficients required for adjusting variates. The adjusted RMGs are independent of one another assuming they follow a joint $n$-variate normal distribution. Residual genetic variation results in correlations among the adjusted RMGs. Regressive models also assume Hardy-Weinberg equilibrium, and the independence of parameter estimation with pedigree size and structure. The likelihoods derived from a normal density function based on randomly sampled pedigrees for these models are given by Bonney (1984), and Demenais \& Bonney (1989). Hypotheses of genetic transmission can be modelled and tested through maximum likelihood procedures.

\section{Analysis and hypothesis testing}

The segregation analysis for this study was performed on an MS-Dos (Microsoft Corp., Redmond, WA) based personal computer. All analyses used the REGC program's class D model of the Statistical Analysis for Genetic Epidemiology (SAGE) Release 2.1 computer software package (Elston, Department of Biometry and Genetics, Louisiana State University Medical Center, New Orleans, LA). It is based on a regressive model, utilizing the method of maximum likelihood to perform pedigree analysis. Optimization of the likelihood functions was achieved through the complete direct search method (Sorant et al., 1992).

Limitations of the algorithm used for segregation analysis in the SAGE program restrict the types of pedigrees that can be analysed (Tran et al., 1991). Pedigrees may range in complexity from a set of fullsibs to an extended pedigree covering many generations and including collateral relatives. However, the program cannot process pedigrees that contain more than one subpedigree or pedigrees with inbreeding or mating loops (Tran et al., 1991). Accordingly, the NCII mating design of the Davis population was subsampled for pedigrees that met the criteria of the SAGE program. Three generations of the population were randomly subsampled to obtain an adequate sample size for CSA. The sample $(n=467$ individuals) included 25 pedigrees, each consisting of a half-sib family, selected such that no two pedigrees were related (relationships were not considered beyond and including first cousin types). The pedigrees ranged in size from seven to 29 individuals, and each contained from two to four parents and offspring.

The parameters available for manipulation in the REGC program to define genetic models are listed in Table 1 . Models were defined by holding some para-
Table 1 List of parameters available in the REGC program used in this investigation

\begin{tabular}{|c|c|}
\hline Parameter & Description \\
\hline$q_{a}$ & $\begin{array}{l}\text { Frequency of allele } a \text { at a single locus with } a \\
\text { and } b \text { alleles }\end{array}$ \\
\hline$\tau_{i}$ & $\begin{array}{l}i=a a, a b \text { or } b b ; \text { transmission probability for } a a, \\
a b \text { and } b b \text { genotypes }\end{array}$ \\
\hline$\mu_{i}$ & $\begin{array}{l}i=a a, a b \text { or } b b ; \text { mean for } a a, a b \text { or } b b \\
\text { genotypes }(i=a a \& a b \text { indicates that both } \\
\text { genotypes have the same mean) }\end{array}$ \\
\hline$\sigma_{\mathrm{rph}}^{2}$ & Residual $^{\mathrm{z}}$ phenotypic variance \\
\hline$\rho_{i}$ & $\begin{array}{l}i=\mathrm{fm}, \text { po or ss; residual }{ }^{\mathrm{z}} \text { correlation between } \\
\text { mates, parent-offspring, and between siblings, } \\
\text { respectively }\end{array}$ \\
\hline
\end{tabular}

${ }^{z}$ residual refers to effects remaining after adjustment for the major gene.

meters constant while obtaining maximum likelihood estimates of the others. The transmission parameters $\left(\tau_{i}\right)$ measure the probabilities that each mating type transmits a specified allele to an offspring. These probabilities under a diploid single locus Mendelian model are 1.0, 0.5 and 0.0 for the $a a, a b$ and $b b$ genotypes, respectively.

Genetic models were tested that included the following sources of variation: major gene, polygenic and environmental effects (arbitrary, dominant and additive models); only major gene effects (Mendelian single locus or MSL model); polygenic and environmental effects (no-major-gene model); and only environmental effects (env. model). The arbitrary model restricts the $\tau_{i}$ to Mendelian probabilities but maximum likelihood estimates are obtained on all other parameters that are included in the complete model; it places no restriction on the location of the mean heterozygote phenotype relative to the homozygote means. The dominant and additive models are similar to the arbitrary model, but assume the heterozygote mean is dependent and appropriately located. The MSL model includes estimates of $\mu_{a a}, \mu_{a b}, \mu_{b b}$ but assumes that the residual correlations among relatives are 0 . The no-major-gene model does not consider the effects of a major locus; therefore the transmission frequencies for each 'genotype' are set equal to the allele frequency $\left(\tau_{i}=q_{a}\right)$, the three types of $\mu_{i}$ replaced with a single population mean, and estimates of the $\rho_{i}$ are obtained. The environmental model likewise sets $\tau_{i}=q_{a}$ and has only a population mean, but $\rho_{\mathrm{po}}$ and $\rho_{\mathrm{ss}}$ are also fixed at 0 .

The likelihood ratio test (LRT), utilizing the statistic $-2(\ln L)$, was used to compare complete with restricted models (Edwards, 1978). The hypothesis that a parameter included in a model is unnecessary is 
tested by subtracting $-2 \ln L$ of the complete model from $-2 \ln L$ of the restricted model, where the complete model obtains maximum likelihood estimates of all the pertinent parameters and the restricted model holds the parameters of interest constant. This difference asymptotically approaches a $\chi^{2}$ distribution with degrees of freedom equal to the difference in the number of independent parameters of each model. Akaike's information criterion (AIC), $-2(\ln L)+2$ (number of parameters), was used to compare nonhierarchical models (Akaike, 1974). The model providing the smallest AIC for a given data set best describes the data based on goodness-of-fit and parsimony.

Including $\tau_{a b}$ in the complete model provides a test for Mendelian transmission when compared with a restricted model that has this parameter externally fixed at 0.5 . A more stringent test obtains estimates of all $\tau_{i}$; however, as the true values for the homozygotes are at the bounds ( 1.0 and 0.0 , respectively), it is often difficult to run this test without these parameters being fixed at the boundaries (McGuffin \& Huckle, 1990). Strategies for inferring genetic control utilizing LRT with pedigree analysis are reviewed in Khoury et al. (1993).
Polygenic variation can be measured by the residual correlation between relatives $\left(\rho_{\mathrm{po}}\right.$ and $\left.\rho_{\mathrm{ss}}\right)$ after the effect of a major gene has been estimated. The residual heritability is calculated as $2 \rho_{\mathrm{po}}$. The $\rho_{\mathrm{ss}}$ estimate is the correlation between full-sibs; full-sib families nested within a half-sib family are considered as independent.

\section{Results}

The normal probability plots for hue are given in Fig. 1 ; each departs from a straight line pattern and follows a sigmoidal-shaped curve. The shapes of the distributions for each generation are similar to the CSA sample distribution $\left(\mu=38.2, \sigma^{2}=335.6\right)$. Therefore, the subsampling procedure did not unduly bias the results. Plants with hues greater than 48 degrees comprise about 20 per cent of the population (Fig. 2); these plants represent one of the modes of the distribution.

A comparison of the genetic models is given in Table 2. The estimates (and SE) of the transmission parameters for the general model were $\tau_{a a}=0.99 \pm 0.03$, $\tau_{a b}=0.53 \pm 0.05$ and $\tau_{b b}=0.26 \pm 0.08$. The analysis indicates that only the arbitrary and dominant models fail to be rejected. The most parsimonious model, as
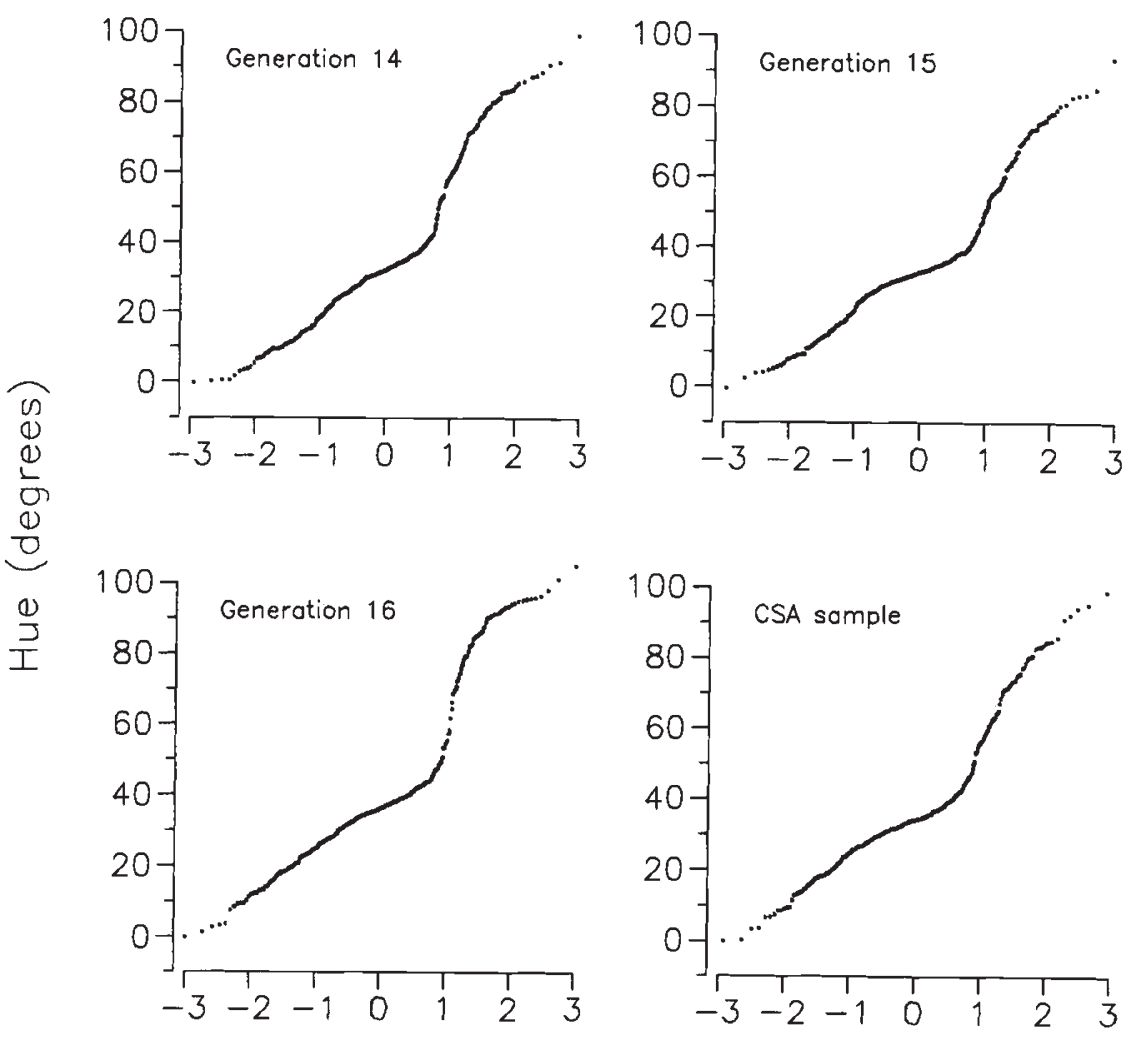

\section{Normal prob. score}

Fig. 1 Normal probability plots of hue for generations $14,15,16$, and the CSA sample of the Davis population of gerbera $(n=438,451,516$ and 467 , respectively). 
Fig. 2 Phenotypic frequency distributions of hue for generations $14,15,16$, and the CSA sample of the Davis population of gerbera.
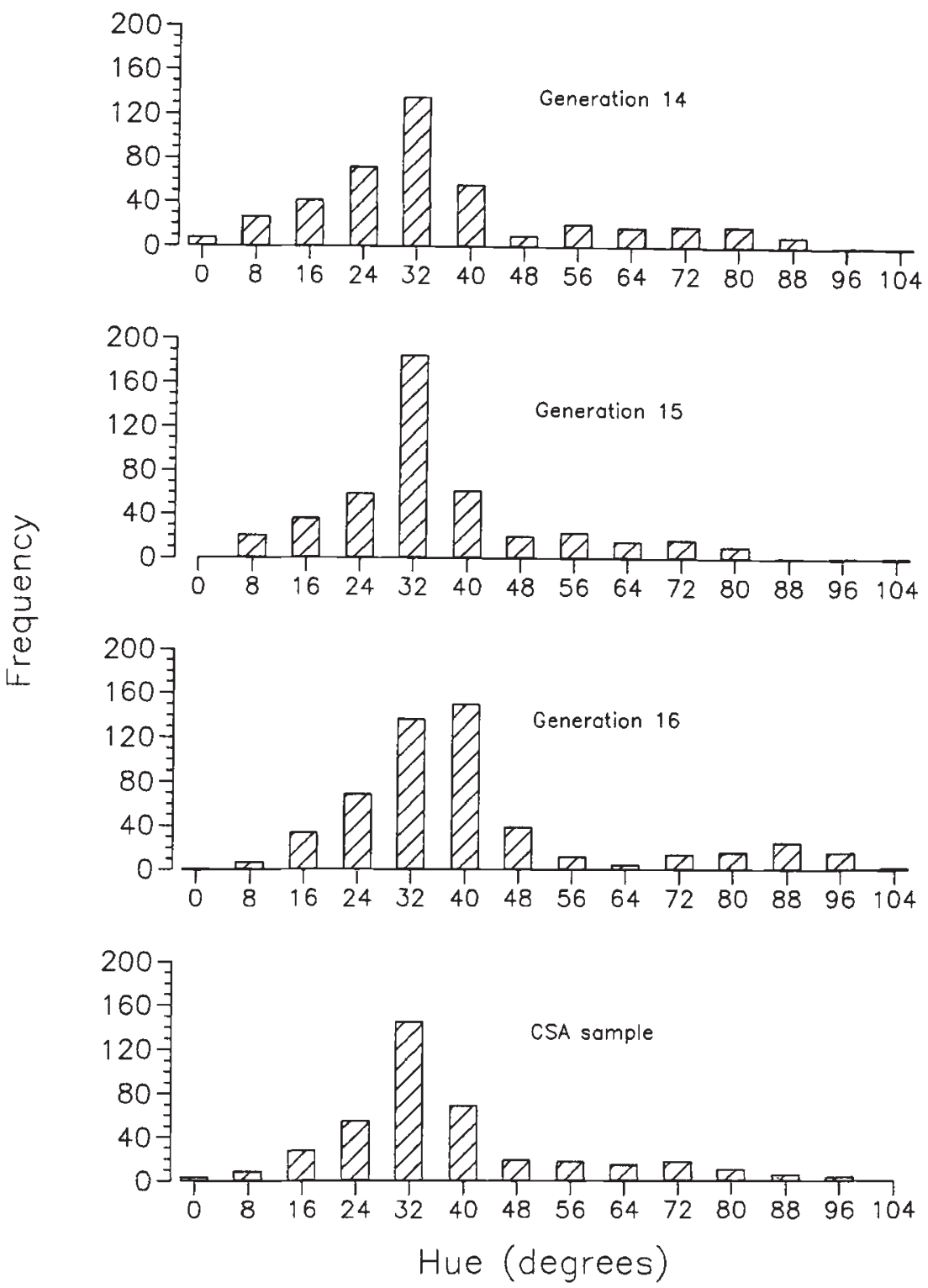

measured by the AIC, was the dominant model (the $a$ allele dominant to the $b$ allele).

The parameter estimates for the dominant model are shown in Table 3. The residual correlation between mates is close to 0 , indicating that mating was random. The putative gene frequency estimate is 0.55 , and the homozygote means are estimated to be 31.5 and 70.5 degrees for the $a$ and $b$ alleles, respectively. Twice the residual parent-offspring correlation is a measure of narrow sense heritability, excluding the effects of the putative major gene (Demenais \& Bonney, 1989). This data set provides an estimate of residual $h^{2}=0.38$.

\section{Discussion}

The sigmoidal patterns displayed in Fig. 1 are diagnostic of samples that represent a mixture of two normal distributions (i.e. bimodality). Genetic relationships among individuals are not used to produce these plots. Therefore, they may be used to support independently the pedigree-based results of CSA (namely the location of the modes and their proportion (Fig. 2) should be consistent with the frequency and mean genotypic value of a gene with major effect detected by CSA).

Except for $\tau_{b b}$, the CSA transmission parameter estimates of the complete model coincide with expected values under a Mendelian hypothesis. The $\tau_{b b}$ estimate may be affected by residual genetic variation, i.e. there may be individuals in the sample homozygous recessive $(b b)$ for the major gene but having a polygenic genotype at other loci producing the dominant phenotype of the major gene. This would cause the $\tau_{b b}$ estimate to deviate from its expected 
Table 2 Likelihood ratio test (LRT) for models of hue in the Davis population of gerbera $(n=467 ; 25$ pedigrees from generations 14,15 and 16)

\begin{tabular}{|c|c|c|c|c|c|c|}
\hline Model & $\begin{array}{l}\text { Parameters } \\
\text { estimated }\end{array}$ & $-2 \ln L$ & d.f. & $\chi^{2}$ & $P$ value & AIC \\
\hline Complete $^{z}$ & $\begin{array}{c}\mu_{a a}, \mu_{a b}, \mu_{b b}, \sigma_{\mathrm{rph}}^{2} \\
\rho_{\mathrm{fm}}, \rho_{\mathrm{po}}, \rho_{\mathrm{ss}}, \tau_{a} \\
\tau_{a a}, \tau_{a b}, \tau_{b b}\end{array}$ & 3753.4 & & & & \\
\hline Arbitrary $^{y}$ & $\mu_{a a}, \mu_{a b}, \mu_{b b}, \sigma_{\mathrm{rph}}^{2}$ & 3754.3 & 3 & 0.86 & 0.835 & 3770.3 \\
\hline Dominant $^{y}$ & $\begin{array}{c}\rho_{\mathrm{fm}}, \rho_{\mathrm{po}}, \rho_{\mathrm{ss}}, q_{a} \\
\mu_{a a}, \mu_{b b}, \sigma_{\mathrm{rph}}^{2}\end{array}$ & 3879.4 & 4 & 0.86 & 0.930 & 3768.3 \\
\hline Additive $^{y}$ & $\begin{array}{c}\rho_{\mathrm{fm}}, \rho_{\mathrm{po}}, \rho_{\mathrm{ss}}, q_{a} \\
\mu_{a a}, \mu_{b b}, \sigma_{\mathrm{rph}}^{2}\end{array}$ & 3879.4 & 4 & 126.04 & $<0.001$ & 3893.4 \\
\hline $\operatorname{MSL}^{x}$ & $\begin{array}{c}\rho_{\mathrm{fm}}, \rho_{\mathrm{po}}, \rho_{\mathrm{ss}}, q_{a} \\
\mu_{a a}, \mu_{a b}, \mu_{b b} \\
\sigma_{\mathrm{rph}}^{2}, \rho_{\mathrm{fm}}, q_{a}\end{array}$ & 3807.7 & 5 & 54.28 & $<0.001$ & 3819.7 \\
\hline $\begin{array}{l}\text { No major } \\
\text { gene }^{w}\end{array}$ & $\begin{array}{c}\mu_{a a}, \sigma_{\mathrm{rph}}^{2}, \rho_{\mathrm{fm}} \\
\rho_{\mathrm{po}}, \rho_{\mathrm{ss}}\end{array}$ & 3949.3 & 6 & 195.90 & $<0.001$ & 3959.3 \\
\hline Env. $^{v}$ & $\mu_{a a}, \sigma_{\mathrm{rph}}^{2}, \rho_{\mathrm{fm}}$ & 4040.4 & 8 & 286.97 & $<0.001$ & 4046.4 \\
\hline
\end{tabular}

$H_{0}$, restricted model; $H_{\mathrm{A}}$, complete model.

See Table 1 for parameter key.

${ }^{\mathrm{z}}$ The complete model estimates of $\tau_{i}$ are $\tau_{a a}=0.99, \tau_{a b}=0.53, \tau_{b b}=0.26$.

${ }^{y}$ Arbitrary, additive and dominant models assume both polygenic and major gene

effects; they differ from one another in the placement of the heterozygote mean.

${ }^{x}$ Mendelian single locus model; no residual correlation among relatives.

${ }^{w}$ Assumes only polygenic and environmental variation.

${ }^{v}$ Assumes only environmental variation, $\rho_{\mathrm{po}}$ and $\rho_{\mathrm{ss}}$ set to zero.

Table 3 Parameter estimates and their standard errors for the dominant genetic model

\begin{tabular}{ccc}
\hline & \multicolumn{2}{c}{ Dominant model } \\
\cline { 2 - 3 } Parameter & Estimate & $\mathrm{SE}$ \\
\hline$q_{a}$ & 0.55 & 0.04 \\
$\mu_{a a \& a b}$ & 31.5 & 0.87 \\
$\mu_{b b}$ & 70.5 & 1.60 \\
$\sigma_{\mathrm{rph}}^{2}$ & 112.6 & 11.21 \\
$\rho_{\mathrm{fm}}$ & 0.04 & 0.126 \\
$\rho_{\mathrm{po}}$ & 0.19 & 0.087 \\
$\rho_{\mathrm{ss}}$ & 0.41 & 0.072 \\
\hline
\end{tabular}

${ }^{z}$ The means for the $a a$ and $a b$ genotypes are constrained to be equivalent in the dominant model.

Mendelian probability. However, this deviation does not cause the general model to provide a better fit than a model with the transmission parameters fixed to Mendelian expectations (for example the arbitrary and dominant models).

The genetic models that exclude either major locus or polygenic effects, or both, are not as satisfactory as models that include these sources of variation (Table 2 ). This is true even when considering the AIC which adds a penalty based on the number of parameters included in a model.

The CSA results are consistent with both the phenotypic distribution of hue and previous studies of pigment chemistry in gerbera. The dominant model provides estimates of $\mu_{a a \& a b}=31.5$ and $\mu_{b b}=71.0$ degrees which is consistent with the interpretation of Fig. 2 as representing a bimodal distribution. The allele frequency of $a$ is 0.55 , consistent with the relative amounts of each mode depicted in Fig. 2 (assuming Hardy-Weinberg equilibrium, the $b b$ genotype frequency is 0.2 ). These two modes correspond to the red and yellow hues, respectively. It has been demonstrated in gerbera that the reds are produced by flavonoid pigments and the yellows by carotenoids (Asen, 1984; Valadon \& Mummery, 1967). These are two distinct biochemical pathways. The putative major gene may be controlling the expression of flavonoid pigments which may mask the expression of carotenoid genes.

Estimates of the phenotypic variance $\left(\sigma^{2}=335.6\right)$ and residual variance $\left(\sigma_{\mathrm{rph}}^{2}=112.6\right)$ of the dominant model provide an estimate of the proportion of the variance accounted for by the major gene, $\sigma_{\mathrm{mg}}^{2}=\sigma^{2}-\sigma_{\mathrm{rph}}^{2}$ (Demenais et al., 1990). The proportion $\sigma_{\mathrm{mg}}^{2} / \sigma^{2}=0.66$, measures the degree of influence 
the putative gene has on hue variability in the Davis population. The remainder of the variance $\left(\sigma_{\mathrm{rph}}^{2}\right)$ is generated by genetic and environmental factors.

The $\rho_{\mathrm{po}}$ and $\rho_{\mathrm{ss}}$ estimates of the dominant model (Table 3 ) both indicate that a substantial amount of residual genetic variation exists. This is also implied by the increase in the AIC of the MSL model (Table 2). The estimate for $\rho_{\mathrm{ss}}$ is significantly greater than the $\rho_{\mathrm{po}}$ because dominance and epistasis inflate the $\rho_{\text {ss }}$ estimate whereas environmental heterogeneity reduces the $\rho_{\mathrm{po}}$ estimate. The environmental heterogeneity may result from measuring parents in different years than their offspring were measured (because the population was grown as an annual with one generation/year).

Complex segregation analysis provides geneticists with the flexibility to define genetic models such that quantitative and qualitative variation can be considered simultaneously. Using pedigree information to interpret patterns of inheritance provides additional flexibility to analyse populations that were not specifically constructed to study complex inheritance. This approach was successful in understanding genetic variation for flower hue in the Davis population of gerbera. However, when applied to the colour attributes chroma and value we were unable to reject the complete model. This may be a consequence of genetic heterogeneity or epistasis for segregating genes of these traits. Genetic heterogeneity results when more than one major gene is segregating for the trait of interest. As the statistical model includes only the effects of one major gene other major genes can distort the transmission probability estimates resulting in lower likelihoods for models constrained to Mendelian transmission probabilities in comparison with the complete model. Apparently, there is a gene segregating for hue in the Davis population with a much larger effect than other genes for this trait, but this differential of effects is diminished for the colour attributes chroma and value.

\section{Acknowledgements}

Fred Bliss, Hongzhan Huang and Douglas V. Shaw provided many helpful comments on earlier versions of this manuscript. We are grateful also to Thomas $\mathrm{R}$. Famula and Alexander F. Wilson for their assistance during this investigation. The results of this paper were obtained by using the program package SAGE, which is supported by a US Public Health Service Resource Grant (1 P41 RRO3655) from the Division of Research Resources.

\section{References}

AKAIKE, H. 1974. A new look at the statistical model identification. IEEE Transactions on Automatic Control AC-19, 716-723.

ASEN, S. 1984. High pressure liquid chromatographic analysis of flavonoid chemical markers in petals from gerbera flowers as an adjunct for cultivar and germplasm identification. Phytochemistry, 23, 2523-2526.

BONNEY, G. E. 1984. On the statistical determination of major gene mechanisms in continuous human traits: regressive models. Am. J. Med. Genet., 18, 731-749.

BURNS, T. L., MOLL, P. P. AND SCHORK, M. A. 1984. Comparisons of different sampling designs for the determination of genetic transmission in quantitative traits. Am. J. Hum. Genet., 36, 1060-1074.

CANNINGS, C., THOMPSON, E. A. AND SKOLNICK, M. H. 1978. Probability functions on complex pedigrees. Adv. Appl. Prob., 10, 26-61.

CHAMBERS, J. M., CLEVELAND, W. S., KLEJNER, B. AND TUKEY, P. A. 1983. Graphical Methods for Data Analysis. Wadsworth International Press, Belmont, CA.

CRANE, M. B., GREENSLADE, R. M., MASSEE, A. M. AND TYDEMAN, H. M. 1936. Studies on the resistance and immunity of apples to woolly aphis, Eriosoma lanigerum (Hausmn.). J. Pomol., 14, 137-163.

DEMENAIS, F. M. AND BONNEY, G. E. 1989. Equivalence of the mixed and regressive models for genetic analysis. I. Continuous traits. Genet. Epidemiol., 6, 597-617.

DEMENAIS, F. M., MURIGANDE, C. AND BONNEY, G. E. 1990. Search for faster methods of fitting the regressive models to quantitative traits. Genet. Epidemiol., 7, 319-334.

EDWARDS, A. W. F. 1978. Likelihood. An Account of the Statistical Concept of Likelihood and its Application to Scientific Inference, 2nd edn. Cambridge University Press, London.

EDWARDS, J. H. 1960. The simulation of Mendelism. Acta Genet. Stat. Med., 10, 63.

ELKIND, Y. AND CAHANER, A. 1986. A mixed model for the effects of single gene, polygenes and their interaction on quantitative traits. I. The model and experimental design. Theor. Appl. Genet., 72, 377-383.

ELKIND, Y., CAHANER, A. AND KEDAR, N. 1990. A mixed model for the effects of single gene, polygenes and their interaction on quantitative traits. 2. The effects of the nor gene and polygenes on tomato fruit softness. Heredity, 64, 205-213.

ELSTON, R. C. AND RAO, D. C. 1978. Statistical modeling and analysis in human genetics. Ann. Rev. Biophys. Bioeng., 7 , 253-286.

ELSTON, R. C. AND STEWART, J. 1971. A general model for the genetic analysis of pedigree data. Hum. Hered., 21, 523-542.

FALCONER, D. S. 1989. Introduction to Quantitative Genetics, 3rd edn, p. 304. Longman Scientific and Technical, New York.

GO, R. C. P., ELSTON, R. C. AND KAPLAN, E. B. 1978. Efficiency and robustness of pedigree segregation analysis. Am. J. Hum Genet., 30, 28-37. 
HARRIS, J. A. 1910. A bimodal variation polygon in Syndesmon thalictroides and its morphological significance. Am. Nat., 44, 19-30.

HOESCHELE, 1. 1988. Statistical techniques for detection of major genes in animal breeding data. Theor. Appl. Genet., 76, 311-319.

ISELIUS, L. 1988. Genetic epidemiology of common diseases in humans. In: Weir, B. S., Eisen, E. J., Goodman, M. M. and Namkoong, G. (eds). Proceedings of the Second International Conference on Quantitative Genetics, 31 May 1987 to 5 June 1987, North Carolina State University, pp. 341-352. Sinauer Associates, Sunderland, MA.

KHOURY, M. J., BEATY, T. H. AND COHEN, B. H. 1993. Fundamentals of Genetic Epidemiology. Oxford University Press, New York.

McGUFFIN, P. AND HUCKLE, P. 1990. Simulation of Mendelism revisited: the recessive gene for attending medical school. Am. J. Hum. Genet., 46, 994-999.

McGUiRE, R. G. 1992. Reporting of objective color measurements. HortScience, 27, 1254-1255.

MITCHELL-OLDS, T. AND BERGELSON, J. 1990. Statistical genetics of an annual plant, Impatiens capensis. I. Genetic basis of quantitative variation. Genetics, 124, 407-415.

POWERS, L. 1950. Gene analysis of weight per locule in tomato hybrids. Bot. Gaz., 112, 163-174.
SINNOTT, E. w. 1932. Shape changes during fruit development in Cucurbita and their importance in the study of shape inheritance. Am. Nat., 66, 301-309.

SORANT, A. J. M., BONNEY, G. E. AND ELSTON, R. C. 1992. Segregation Analysis of a Continuous Trait Under Approximations to Class $A, B, C$, and $D$ Regressive Models (REGC ver. 4.0.01). Louisiana State University Medical Center, New Orleans, LA.

SMITH, C. A. B. 1976 . The use of matrices in calculating Mendelian probabilities. Ann. hum. Genet., 40, 37-54.

TOURJEE, K. R., HARDING, J. AND BYRNE, T. G. 1993. Colorimetric analysis of gerbera flowers. HortScience, 28, 735-737.

TRAN, L. D., ELSTON, R. C., KEATS, B. J. B. AND WILSON, A. F. 1991. Family Structure Program User's Manual. Louisiana State University Medical Center, New Orleans, LA.

VALADON, L. R. G. AND MUMMERY, R. S. 1967. Carotenoids of certain Compositae flowers. Phytochemistry, 6, 983-988.

VANDON1, G. C. 1977. Genetic analysis of some phenotypes of Gerbera jamesoni. Genet. Agr., 31, 113-119.

YU, Y., BYRNE, T. AND HARDING, J. 1991. Quantitative genetic analysis of flowering time in the Davis population of gerbera. I. Components of genetic variance and heritability. Euphytica, 53, 19-23. 